\title{
ALMOST EVERYWHERE CONVERGENCE OF POISSON INTEGRALS ON GENERALIZED HALF-PLANES
}

\author{
BY NORMAN J. WEISS ${ }^{1}$
}

\author{
Communicated by A. P. Calderon, December 6, 1967
}

1. Introduction. A classical theorem of Fatou states that if $f$ is an $L^{p}$ function on the line (circle), $p \geqq 1$, and if the harmonic function $F$ on the upper half-plane (disk) is the Poisson integral of $f$, then $F(z)$ $\rightarrow f(x)$ as $z \rightarrow x$ nontangentially for a.e. $x$ on the line (circle).

Generalizations in several directions have recently been found, e.g. [1], [2], [4], [6]. Our result, stated precisely below, is Fatou's theorem for generalized upper half-planes holomorphically equivalent to bounded symmetric domains and functions of type $L^{p}, p>1$, or locally of type $L \log +L$. Details will appear elsewhere.

In $\$ 2$, we sketch the setting and state our result explicitly. The proof is case-by-case, and includes the case of the exceptional domains; $\$ 3$ is devoted to a sketch of the proof in a typical case.

2. The theorem. Let $D$ be a generalized upper half-plane, i.e.

$$
D=\left\{(z, w) \in V_{1} \times V_{2}: \operatorname{Im} z-\Phi(w, w) \in \Omega\right\},
$$

where $V_{1}$ is a complex vector space with a given real form, $V_{2}$ is a complex vector space, $\Omega \subset \operatorname{Re} V_{1}$ is an open cone, and $\Phi: V_{2} \times V_{2} \rightarrow V_{1}$ is hermitian symmetric bilinear with respect to $\operatorname{Re} V_{1}$ such that $\Phi(w, w) \in \bar{\Omega}$. When $\Omega$ is a domain of positivity and $\Phi$ satisfies certain symmetry and homogeneity properties, $D$ is holomorphically equivalent to a bounded symmetric domain [5]. The distinguished boundary of $D$ is

$$
B=\{(z, w): \operatorname{Im} z-\Phi(w, w)=0\} .
$$

We identify $B$ with $\operatorname{Re} V_{1} \times V_{2}$ by associating to $(x+i \Phi(w, w)$, w) the pair $(x, w)$. There is a nilpotent group $\mathfrak{N}$ of automorphisms of $D$ which acts transitively on $B$ and is also equal to $\operatorname{Re} V_{1} \times V_{2}$ as a set. Haar measure on $\mathfrak{R}$ is the induced Euclidean measure.

The Poisson kernel, $P(u, \zeta)$, is defined on $B \times D$, and the Poisson integral of a function $f$ on $B$ is

$$
F(\zeta)=\int_{B} f(u) P(u, \zeta) d u .
$$

1 Partially supported by the U. S. Air Force Office of Scientific Research; Harmonic Analysis Contract at Princeton University. 
For details of the above, see [3].

For $u \in B, t \in \Omega$ we write $u_{t}=u+(i t, 0)$. Also, let $I$ be the base point in $\Omega$.

THEOREM 1. Let $D$ be a generalized upper half-plane holomorphically equivalent to a bounded symmetric domain. Suppose that $f \in L^{p}(B)$, $p>1$, or that $f \in L \log ^{+} L$ locally and is bounded off a bounded set. Then

$$
\lim _{r \rightarrow 0} F\left(u_{\tau I}\right) \rightarrow f(u) \text { for almost every } u \in B .
$$

There are more general types of convergence. We say that $t \in \Omega$ approaches 0 restrictedly if $t$ is constrained to lie in a proper subcone of $\Omega$. And we say that $u_{t} \rightarrow u_{0}=g_{0} \cdot 0$ admissibly if $u_{t}$ stays in some

$$
\Gamma_{\alpha}\left(u_{0}\right)=\left\{g_{0} g \cdot(i t, 0): g=(a, c), \max \left\{|a|,|c|^{2}\right\}<\alpha|t|\right\} .
$$

THEOREM 2. Under the hypothesis of Theorem 1, $F\left(u_{t}\right) \rightarrow f\left(u_{0}\right)$ for a.e. $u_{0} \in B$ as $u_{t} \rightarrow u_{0}$ admissibly and restrictedly.

3. The proof. The proof for domains $D$ which are tube domains, i.e. for which $V_{2}=0$, is contained in [6]. The remaining domains, with the exception of one of dimension 16, fall into two large classes, type I and type IIIb. We indicate the proof of Theorems 1 and 2 for domains of type $I$. The complete proof requires only slight modification.

There is a domain of type $I$ for each pair of integers $n, m, n>0$, $m \geqq 0$. As a bounded domain, it is realized as the space of complex $n \times(n+m)$ matrices $\zeta$ satisfying $\zeta \zeta^{*}<I$. In the realization we consider, $V_{1}$ is the complexification of the real vector space of hermitian symmetric $n \times n$ matrices, $V_{2}$ is the space of complex $n \times m$ matrices, $\Omega$ is the cone of positive definite matrices, and $\Phi\left(w, w_{1}\right)=w_{1}^{*}$. Thus

$$
D=D_{n, m}=\left\{(x+i y, w): y-w w^{*}>0\right\} .
$$

The Poisson integral $F\left((g \cdot 0)_{t}\right)$ is shown to be dominated by a sum of maximal functions $f^{*}{ }_{(j)(k)}(g), g \in \mathfrak{R}$. We define these.

Let $(j)$ and $(k)$ be, respectively, $n$-tuples and $m$-tuples of nonnegative integers and $t>0$.

$$
\begin{aligned}
& R_{(j)}^{t}=\left\{r=\left(r_{1}, \cdots, r_{n}\right) \in E_{n}:\left|r_{i}\right| \leqq 2^{j_{i}} t\right\}, \\
& S_{(k)}^{t}=\left\{s \in E_{m}:\left|s_{i}\right| \leqq 2^{k_{i}} t\right\} .
\end{aligned}
$$

Every $x \in \operatorname{Re} V_{1}$ can be written in the form $x=k^{-1} d(r) k, k \in U(n)$, $r \in E_{n}$, where $d(r)$ is the diagonal $n \times n$ matrix whose entries are the $r_{i}$. And every $w \in V_{2}$ can be written $w=u \tilde{d}(s) v, u \in U(n), v \in U(m)$, 
$s \in E_{m}$, where $\tilde{d}(s)$ is the $n \times m$ diagonal-form matrix whose entries are the $s_{i}$. We set

$$
B_{(j)(k)}^{t}=\left\{(x, w)=\left(k^{-1} d(r) k, u \tilde{d}(s) v\right): r \in R_{(j)}^{t}, s \in S_{(k)}^{t^{1 / 2}}\right\} .
$$

(If $m \geqq n$, the rectangles $S$ lie in $E_{n}$, but this makes no difference.)

There is defined for each $(j)$ and $(k)$ a sequence of neighborhoods

$$
U(n)=N_{(j)(k) 0} \supset N_{(j)(k) 1} \supset \cdots \supset\{I\},
$$

and we define

$$
\begin{gathered}
E_{(j)(k) l}^{t}=\left\{(x, w)=\left(k^{-1} d(r) k, u \tilde{d}(s) v\right) \in H_{(j)(k)}^{t}: k u \in N_{(j)(k) l}\right\} \\
f_{(j)(k) l}^{*}(g)=\sup _{t>0}\left|E_{(j)(k) l}^{t}\right|^{-1} \int_{E_{(j)(k) l}} f(g h) d h .
\end{gathered}
$$

We abuse notation now by writing $t$ for both a positive number and the matrix $t I$.

LEMMA 1.

$$
\sup _{l>0} F\left((g \cdot 0)_{t}\right) \leqq A \sum_{(j)(k)} 2^{-[(1 / 2)|j|+|k|]} \sum_{l=1}^{L} f_{(j)(k) l}^{*}(g),
$$

where $L$ depends only on $m$ and $n$.

Proof. It is enough to prove the inequality when $g=0$. We notice that

$$
P((x, w),(i t, 0))=P_{t}(x, w)=\left\{\frac{\operatorname{det} t}{\left|\operatorname{det}\left(x+i\left[w 2 w^{*}+t\right]\right)\right|^{2}}\right\}^{n+m} .
$$

The method of proof is to compare the size of $P_{t}(u), u \in E^{t}{ }_{(j)(k) l-1}$ $-E_{(j-1)(k-1) l}^{b}$, with the size of $\left|E_{(j)(k) l}\right|$. (The $t^{1 / 2}$ factor in the definition of $E_{(j)(k) l}$ is due to the ww* term in (1).) The necessity for considering the neighborhoods $N_{0} \supset N_{1} \supset \cdots$ may be seen by considering a special case.

In particular, if $x=k^{-1} d\left(2^{j}, 0, \cdots, 0\right) k, w=u \tilde{d}\left(2^{j / 2}, 0, \cdots, 0\right) v$, then $\left|\operatorname{det}\left(x+i\left[w w^{*}+I\right]\right)\right|^{2}$ depends on $k u$, ranging from $2^{2 j}+\left(2^{j}+1\right)^{2}$ when $k u=I$ to $\left(2^{2 j}+1\right)\left(2^{j}+1\right)^{2}$. The proof that the number of $N_{j}$ that need be considered is finite involves an induction, and is complicated.

The proof of Theorem 1 is now routine once one establishes

LEMma 2. $\left\|f_{(j)(k) l}^{*}\right\|_{p} \leqq A_{p}\|f\|_{p}$, where $A_{p}$ is independent of $(j),(k)$ and $l$. 
Proof. It is not hard to show that

(2) $f_{(j)(k) l}^{*}(g) \leqq A \frac{\int_{U(n)} \int_{U(n)} \int_{U(m)} \bar{f}_{(j)(k)}(g ; k, u, v) \chi(k u) d v d u d k}{\iiint \chi(k u) d v d u d k}$, where $\chi$ is the characteristic function of $N_{(j)(k) l}$ and $f_{(j)(k)}(g ; k, u, v)$

$$
=\sup _{i>0}\left|R_{(j)}^{t}\right|^{-1}\left|S_{(k)}^{t^{1 / 2}}\right|^{-1} \int_{R_{(j)}^{t}} \int_{S_{(k)}^{t^{1 / 2}}} f\left(g \cdot\left(k^{-1} d(r) k, u \tilde{d}(s) v\right)\right) d s d r .
$$

The function $\bar{f}(\cdot ; k, u, v)$ may be thought of as giving maximal averages over $m+n$-dimensional rectangles "pointed" in the direction determined by $u, v$ and $k$. Now the subgroup

$$
\mathfrak{S}_{k, u, v}=\left\{h=\left(k^{-1} d(r) k, u \tilde{d}(s) v\right): r \in E_{n}, s \in E_{m}\right\}
$$

of $\mathfrak{N}$ is isomorphic to $E_{n} \times E_{m}$, and so $\bar{f}_{(j)(k)}$ restricted to the coset $\mathrm{g} \cdot \mathfrak{S}_{k, u, v}$ is an ordinary maximal function. Thus

$$
\int_{\mathfrak{W}_{k, u, v}}\left|f_{(j)(k)}(g h ; k, u, v)\right|^{p} d h \leqq B_{p} \int_{\mathfrak{W}_{k, u, v}}|f(g h)|^{p} d h .
$$

Integrating over $\mathfrak{N} / \mathfrak{W}$ on both sides of (3), one has

$$
\int_{\mathfrak{R}}|\bar{f}(g ; k, u, v)|^{p} d g \leqq B_{p} \int_{\mathfrak{N}}|f(g)|^{p} d g .
$$

This, together with (2), proves the lemma.

The proof that $\left\|f_{(j)(k))}^{*}\right\|_{1} \leqq A_{1}\|f\|_{L \log }{ }_{L}$ depends on the analogous result for ordinary maximal functions. To prove Theorem 1 in the case $f \in L^{1}$ would involve establishing a weak-type inequality,

$$
\left|\left\{g:\left|f_{(j)(k) l}^{*}(g)\right|>s\right\}\right|<A_{0} s^{-1}\|f\|_{1} .
$$

Since the weak-type inequality for rectangular maximal functions cannot be "rotated" the way norm inequalities can, further analysis is necessary. This analysis has been performed by E. M. Stein (see [7]) and the author; and will appear.

We conclude by noting that Theorem 2 is a consequence of Theorem 1 and the following result, which is a slight extension of the corresponding result in the tube domain case. 
LeMmA 3. Suppose that $u_{t} \rightarrow u_{0}$ restrictedly and admissibly. Let $\bar{t}>0$ be the smallest eigenvalue of $t$. Then, for any $u^{\prime} \in B$,

$$
P\left(u^{\prime}, u_{t}\right) \leqq A P\left(u^{\prime},\left(u_{0}\right)_{t I}\right) \text {. }
$$

\section{REFERENCES}

1. S. Helgason and A. Koranyi, A Fatou-type theorem for harmonic functions on symmetric spaces, Bull. Amer. Math. Soc. 74 (1968), 258-263.

2. A. Knapp, Fatou's theorem for symmetric spaces. I, Mimeographed notes, Cornel] Univ., Ithaca, N. Y., 1967.

3. A. Koranyi, The Poisson integral for generalized half-planes and bounded symmetric domains, Ann. of Math. (2) 82 (1965), 332-350.

4. A. Koranyi and E. M. Stein, Fatou's theorem for generalized halfplanes, C.I.M.E. Summer Course on Bounded Homogeneous Domains, Cremonese, 1967.

5. A. Koranyi and J. Wolf, Realization of hermitian symmetric spaces as generalized half-planes, Ann. of Math. (2) 81 (1965), 265-288. .

6. N. J. Weiss, Almost everywhere convergence of Poisson integrals on tube domains over cones, Trans. Amer. Math. Soc. 129 (1967), 283-307.

7. E. M. Stein, Maximal functions and Fatou's theorem, C.I.M.E. Summer Course on Bounded Homogeneous Domains, Cremonese, 1967.

Princeton University 